\title{
Correction to: Exploiting hyperbolic metamaterial as a substrate for graphene surface plasmonic Cherenkov THz radiation source
}

\author{
Nalini Pareek ${ }^{1,2} \cdot$ Niladri Sarkar ${ }^{3} \cdot$ Anirban Bera $^{1,2}$
}

Published online: 6 January 2022

(c) The Author(s), under exclusive licence to Springer-Verlag GmbH, DE part of Springer Nature 2022

Correction to: Appl Phys A (2020) 126:882

https://doi.org/10.1007/s00339-020-04064-6

In this article, the affiliation 2 for Nalini Pareek and Anirban

Bera was incorrectly given.

The correct affiliation is as follows.

Academy of Scientific and Innovative Research, (AcSIR), Ghaziabad 201002, India.

Publisher's Note Springer Nature remains neutral with regard to jurisdictional claims in published maps and institutional affiliations.

The original article can be found online at https://doi.org/10.1007/ s00339-020-04064-6.

Nalini Pareek

np@ceeri.res.in

1 CSIR-Central Electronics Engineering Research Institute, Pilani 333031, India

2 Academy of Scientific and Innovative Research (AcSIR), Ghaziabad 201002, India

3 Birla Institute of Technology \& Science, Pilani, Rajasthan 333031, India 